\title{
RESEARCH
}

Open Access

\section{Phospho-Tyr705 of STAT3 is a therapeutic target for sepsis through regulating inflammation and coagulation}

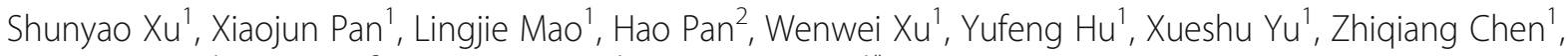
Songzan Qian ${ }^{1}$, Yincai Ye ${ }^{3}$, Yueyue Huang ${ }^{1}$ and Jingye Pan $^{1^{*}}$ (D)

\begin{abstract}
Background: Sepsis is an infection-induced aggressive and life-threatening organ dysfunction with high morbidity and mortality worldwide. Infection-associated inflammation and coagulation promote the progression of adverse outcomes in sepsis. Here, we report that phospho-Tyr705 of STAT3 (pY-STAT3), not total STAT3, contributes to systemic inflammation and coagulopathy in sepsis.

Methods: Cecal ligation and puncture (CLP)-induced septic mice were treated with BP-1-102, Napabucasin, or vehicle control respectively and then assessed for systemic inflammation, coagulation response, lung function and survival. Human pulmonary microvascular endothelial cells (HPMECS) and Raw264.7 cells were exposed to lipopolysaccharide (LPS) with pharmacological or genetic inhibition of pY-STAT3. Cells were assessed for inflammatory and coagulant factor expression, cell function and signaling.

Results: Pharmacological inhibition of pY-STAT3 expression by BP-1-102 reduced the proinflammatory factors, suppressed coagulation activation, attenuated lung injury, alleviated vascular leakage and improved the survival rate in septic mice. Pharmacological or genetic inhibition of pY-STAT3 diminished LPS-induced cytokine production in macrophages and protected pulmonary endothelial cells via the IL-6/JAK2/STAT3, NF-KB and MAPK signaling pathways. Moreover, the increase in procoagulant indicators induced by sepsis such as tissue factor (TF), the thrombin-antithrombin complex (TAT) and D-Dimer were down-regulated by PY-STAT3 inhibition.
\end{abstract}

Conclusions: Our results revealed a therapeutic role of PY-STAT3 in modulating the inflammatory response and defective coagulation during sepsis.

Keywords: Sepsis, STAT3, Inflammation, Coagulation

\footnotetext{
*Correspondence: wmupanjingye@126.com

'Department of Intensive Care Unit, The First Affiliated Hospital of Wenzhou Medical University, Nanbaixiang road, Wenzhou, Zhejiang 325000, P.R. China Full list of author information is available at the end of the article
}

(C) The Author(s). 2020 Open Access This article is licensed under a Creative Commons Attribution 4.0 International License, which permits use, sharing, adaptation, distribution and reproduction in any medium or format, as long as you give appropriate credit to the original author(s) and the source, provide a link to the Creative Commons licence, and indicate if changes were made. The images or other third party material in this article are included in the article's Creative Commons licence, unless indicated otherwise in a credit line to the material. If material is not included in the article's Creative Commons licence and your intended use is not permitted by statutory regulation or exceeds the permitted use, you will need to obtain permission directly from the copyright holder. To view a copy of this licence, visit http://creativecommons.org/licenses/by/4.0/ The Creative Commons Public Domain Dedication waiver (http://creativecommons.org/publicdomain/zero/1.0/) applies to the data made available in this article, unless otherwise stated in a credit line to the data. 


\section{Background}

Sepsis, a major burden on public health, is defined as a life-threatening organ dysfunction that results from a dysregulated host response to infection [1-3]. Despite advances in management in the past decades, sepsis is still the main cause of death in intensive care units with limited therapeutic opinions [4-7].

It is widely known that inflammation and coagulation are involved in the pathogenesis of sepsis in a mutually promoting manner [8-10]. Macrophages and endothelial cells play critical roles as responsive cells in aspects of inflammatory response and coagulative function during sepsis [11, 12]. A large number of studies have established that proinflammatory factors, such as IL-1 $\beta$, IL-6 and TNF- $\alpha$, function as mediators of the procoagulant process $[13,14]$. The excessive cytokines from activated monocytes and endothelial cells immediately give rise to substantial release of TF that initiates the coagulation pathway, which in return leads to aggravated systemic inflammatory responses and tissue injury [15-17]. In addition, endothelial cells are in an unstable state with inflammatory mediators and innate immune elements and coagulation systems in sepsis [18]. The barrier function of endothelial cells is impaired which may result in adverse outcomes [19-21].

The IL-6/JAK2/STAT3 pathway is a major signaling pathway involved in modulating the inflammatory response during the pathogenesis of disease [22]. IL-6 is a pivotal cytokine with diverse physiological functions and increasing IL-6 level is one of the hallmarks of sepsis [23]. Moreover, IL-6 is reported to promote an increase in endothelial permeability in the inflammatory response [24]. Previous studies have shown that JAK2 and STAT3 are activated in an experimental mammalian sepsis model $[25,26]$. The JAK2 inhibition can protect the animals from polymicrobial sepsis by modulating macrophage activation and cytokine production [27]. The suppression of STAT3 activity ameliorates lung inflammatory responses in LPS-induced acute lung injury (ALI) [28, 29]. However, mice with a conditional deletion of STAT3 in macrophages or endothelial cells are susceptible to LPS-induced septic shock associated with increased production of cytokines and adverse survival [30, 31]. To determine the intrinsic mechanism, we designed in vitro experiments utilizing macrophages and endothelial cells and in vivo experiments with cecal ligation and puncture (CLP)-induced septic mice. As a result, our findings provide evidence that inhibition of phospho-Tyr705 of STAT3 (pYSTAT3), not total STAT3, can markedly limit the hyperactivation of the inflammatory response, suppress coagulation activation, protect endothelial barrier function, alleviate lung injury and improve the survival in septic mice.

\section{Methods}

\section{Reagents and antibodies}

LPS was purchased from Sigma (Escherichia coli O111: B4, L2630). BP-1-102 (BP) was from MedChemExpress (HY-100493) and Napabucasin (Na) was purchased from Topscience (T3218). Starch broth was purchased from Sigma (P0727). The following antibodies for western blotting and immunofluorescence were used: antiSTAT3 (CST, \#9139), anti-p-STAT3 (CST, \#9145), antiERK1/2 (CST, \#4695), anti-p-ERK1/2 (CST, \#4370), anti- $\beta$-actin (CST, \#3700), anti-TF (Abcam, \#ab151748), anti-PAR1 (Santa Cruz, \#sc5605), anti-MMP9 (Proteintech, \#10375-2-AP), anti-Ki67 (Abcam, \#ab16667), antiF4/80 (Santa Cruz, \#sc26642), anti-VE-cadherin (Abcam, \#ab33168), anti- $\alpha$-E-catenin (Santa Cruz, \#sc9988), antic-Jun (CST, \#9165), anti-p-JNK (CST, \#9255), anti-P38 (CST, \#8690), anti-p-P38 (CST, \#9216), anti-AKT (CST, \#4691), anti-p-AKT (CST, \#4060), anti-P65 (CST, \#9936), anti-p-P65 (CST, \#9936), anti-JAK2 (CST, \#3230), anti-p-JAK2 (CST, \#3776), anti-TNF- $\alpha$ (CST, \#3707), anti-p-IKK $\alpha / \beta$ (CST, \#9936), anti-IKK $\beta$ (CST,

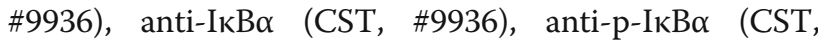
\#9936), goat anti-rabbit HRP-conjugated polyclonal antibody (Bio-Rad, \#1706515), goat anti-mouse HRPconjugated polyclonal antibody (Bio-Rad, \#1706516), anti-rabbit FITC (Abcam, \#ab6717), anti-mouse PE (Abcam, \#ab130774), anti-rabbit Alexa Fluor (Abcam, \#ab150078) and DAPI (CST, \#4083). The following ELISA kits were used: TNF- $\alpha$ (Multi Sciences, \#EK282), IL-1 $\beta$ (Abcam, \#197742), IL-6 (Multi Sciences, \#EK206), CXCL10 (Multi Sciences, \#EK268), TF (Abcam, \#ab214091), PAI1 (Westang, \#F11404), TAT (Westang, \#F11582), D-Dimer (Westang, \#F10354) and VEcadherin (Abcam, \#210968).

\section{Animals}

Toll-like receptor 4 (TLR4) mutant male C57BL/10ScNJ mice (TLR ${ }^{\text {mut }}$ ) were purchased from the Jackson Laboratory. Male C57BL/6 mice (8-10 weeks old) were purchased from Shanghai SLAC Laboratory Animal Co (Shanghai, China). All mice were maintained in a pathogen-free facility under an automated $12 \mathrm{~h}$ darklight cycle at an ambient temperature of $23 \pm 3{ }^{\circ} \mathrm{C}$ and a relative humidity of $55 \pm 10 \%$. Food and water were available ad lib. We conducted all animal care and experimentation with approval from Wenzhou Medical University Institutional Animal Care and Use Committees.

\section{Cecal ligation and puncture (CLP)}

The sepsis mouse model was induced by CLP as previously described [32]. The mice were anesthetized with $1 \%$ sodium pentobarbital $(0.1 \mathrm{ml} / 10 \mathrm{~g}$ body weight; Solarbio, Beijing, China) before the operation. After an 
abdominal incision, the cecum was identified, ligated at the terminal part, and punctured twice with a 21-gauge needle (Kindly, Shanghai, China) to gently squeeze a droplet of its feces. Then the cecum was returned to the abdominal cavity and the abdomen was sutured in two layers. A sham group was established similarly without ligation or puncture. Subsequently, all the mice were subcutaneously injected with $1 \mathrm{ml}$ prewarmed saline for fluid resuscitation. BP $(5 \mathrm{mg} / \mathrm{kg})$ and $\mathrm{Na}(10 \mathrm{mg} / \mathrm{kg})$ dissolved in vehicle (2.5\% DMSO, 2.5\% Tween-80 and 95\% PBS) were administered intraperitoneally to mice $2 \mathrm{~h}$ before CLP (optimal dosage of inhibitors was measured previously). The CLP group was just intraperitoneally treated with vehicle as a control. The animals were closely assessed every $6 \mathrm{~h}$ for the following 4 days and euthanized at the moribund stage. Plasma samples and lung tissues were collected $24 \mathrm{~h}$ after CLP.

\section{ELISA analyses}

The supernatant from in vitro cultured cells or the plasma from experimental mice was quantified using ELISA kits following the manufacturer's protocols.

\section{Cell culture and stimulation}

The murine macrophage cell line Raw264.7 (used within 10 passages) was purchased from American Type Culture Collection (ATCC) and cultured in Dulbecco's modified Eagle's medium (Gibco, Life Technologies, Germany) supplemented with $10 \%$ fetal bovine serum (FBS) (Sigma-Aldrich, St. Louis, MO, USA) and 1\% penicillin-streptomycin (Solarbio, Beijing, China) at $37^{\circ} \mathrm{C}$ in a $5 \% \mathrm{CO} 2$ humidified chamber. Human pulmonary microvascular endothelial cells (HPMECs) were purchased from ScienCell Research Laboratories (Carlsbad, CA, USA) and cultured in the specialized medium (ScienCell, USA). Raw264.7 cells and HPMECs were pretreated with BP-1-102 (5 $\mu \mathrm{M}$, dissolved in DMSO) for 2 $\mathrm{h}$ and then stimulated with LPS $(1 \mu \mathrm{g} / \mathrm{ml})$ for 0.5 or $6 \mathrm{~h}$.

Peritoneal macrophages (PMs) were isolated from 6to 7 -week-old C57BL/10ScNJ and C57BL/6 mice as previously described [32]. Briefly, mice were intraperitoneally injected with $2 \mathrm{ml}$ of starch broth (Sigma, USA), and then the peritoneal cavity was washed with RPMI medium (Gibco, Life Technologies, Germany) three times to isolate peritoneal exudate cells. After $2 \mathrm{~h}$ of culture, the adhered cells were used as macrophages. PMs were stimulated with LPS $(1 \mu \mathrm{g} / \mathrm{ml})$ for $6 \mathrm{~h}$ for further experiments.

\section{Lentivirus transfection}

To genetically suppress phospho-Tyr705 of STAT3 (pYSTAT3), the Y705 phosphorylation STAT3 mutant (Y705A) in which Y705 was replaced with alanine was generated from the GV492 vector (GeneChem,
Shanghai, China) containing Ubi-MCS-3FLAG-CBhgcGFP-IRES-puromycin. The Raw264.7 cells were transfected following the manufacturer's instructions (GeneChem, Shanghai, China).

\section{Tube formation assay}

HPMECs that reached $80 \%$ confluency were pretreated with BP $(5 \mu \mathrm{M}$, dissolved in DMSO $)$ or DMSO as a control. After $2 \mathrm{~h}$, the cells $\left(15 \times 10^{4} /\right.$ well $)$ were digested with trypsin (0.25\% with EDTA, Gibco) and respectively seeded in 24-well plates covered with $120 \mu$ l polymerized Matrigel (BD Biosciences). Then LPS $(1 \mu \mathrm{g} / \mathrm{ml})$ was added for $2 \mathrm{~h}$, and images were collected with a light microscope.

\section{HPMECs permeability assay}

HPMECs $\left(20 \times 10^{4} /\right.$ well $)$ were seeded in the upper chamber of 24 -well Tanswell plate $(0.4 \mu \mathrm{m}$ pore; NEST Biotechnology, Jiangsu). The cells were cultured until confluence and then pretreated with BP for $2 \mathrm{~h}$. LPS $(1 \mu \mathrm{g} / \mathrm{ml})$ was added to the apical medium for $6 \mathrm{~h}$. The medium supernatant of each group was collected. The apical medium was then replaced with $200 \mu \mathrm{l}$ of HRP solution $(50 \mathrm{ng} / \mathrm{ml})$. After incubation for $1 \mathrm{~h}$, the medium of the lower chamber was collected and the penetrating HRP was assessed using a colorimetric assay with Ophenylenediamine for $10 \mathrm{~min}$ incubation at $37^{\circ} \mathrm{C}$. The $\mathrm{OD}_{405}$ was measured by a microplate reader (Molecular Devices, Hercules, CA, USA). To check the indirect effect of the cytokines on junctions, we transferred the collected medium supernatant to the corresponding HPMECs that recently reached confluency for $6 \mathrm{~h}$ and then assessed cell permeability as described above.

\section{Cell counting Kit-8 (CCK-8) assay}

A CCK-8 kit (Dojindo, Japan) was used to analyze the cell viability. Raw264.7 cells or HPMECs were seeded at 3000 cells per well in a 96-well plate and given the corresponding treatment. After $24 \mathrm{~h}, 20 \mu \mathrm{l}$ of CCK8 solution was added for $2 \mathrm{~h}$ incubation at $37^{\circ} \mathrm{C}$. $\mathrm{OD}_{450}$ values were measured.

\section{Immunoblotting}

Immunoblotting analysis was carried out as we previously described [32]. The ECL Chemiluminescent Reagent (Advansta, USA) was used to visualize the proteins and the bands were quantitated by Image (NIH, USA).

\section{RT-PCR}

Total RNA was extracted from cultured cells and lung tissues using the TRIzol reagent (Invitrogen, CA). Moloney murine leukemia virus reverse transcriptase (Invitrogen, USA) was used for cDNA synthesis according to the manufacturer's protocols. The LightCycler (Roche 
Table 1 Primer sequences for RT-PCR

\begin{tabular}{|c|c|c|}
\hline Gene & Forward primer & Reverse primer \\
\hline Mouse GAPDH & GCACAGTCAAGGCCGAGAAT & GCCTTCTCCATGGTGGTGAA \\
\hline Mouse IL-1 $\beta$ & TGCCACCTITGACAGTGATG & TTCTTGTGACCCTGAGCGAC \\
\hline Mouse IL-6 & GCCTTCTTGGGACTGATGCT & TGTGACTCCAGCTTATCTCTTGG \\
\hline Mouse IL-10 & TAAGGCTGGCCACACTTGAG & GTITCAGGGATGAAGCGGC \\
\hline Mouse TNF-a & ACCCTCACACTCACAAACCA & ACCCTGAGCCATAATCCCCT \\
\hline Mouse CXCL1 & GGATGCCACAGGATTCCATA & GTGCCATCAGAGCAGTCTGT \\
\hline Mouse CXCL10 & CCAAGTGCTGCCGCTCATTTTC & TCCCTATGGCCCTCATTCTCA \\
\hline Mouse MCP1 & TAAAAACCTGGATCGGAACCAAA & GCATTAGCTTCAGATTTACGGGT \\
\hline Mouse COX2 & TGCACTATGGTTACAAAAGCTGG & TCAGGAAGCTCCTTATTTCCCTT \\
\hline Mouse MMP1 & TGTTTGCAGAGCACTACTTGAA & CAGTCACCTCTAAGCCAAAGAAA \\
\hline Mouse MMP8 & TGGTGATTTCTTGCTAACCCC & TACACTCCAGACGTGAAAAG \\
\hline Mouse MMP9 & GGACCCGAAGCGGACATTG & CGTCGTCGAAATGGGCATCT \\
\hline Mouse ICAM1 & TTCTCATGCCGCACAGAACT & TCCTGGCCTCGGAGACATTA \\
\hline Mouse TF & GCCACCATCTITATCATCCTCC & AGCCTTTCCTCTATGCCAAGC \\
\hline Mouse PAR1 & CGCAGCGTITACGGGAAC & CTGGATCGGATACACCACCG \\
\hline Mouse tPA & AGATGAGCCAACGCAGACAA & AACTTCGGACAGGCACTGAG \\
\hline Mouse PAl1 & GACGTTGTGGAACTGCCCTA & TCGCTATTGGGCCACCATTT \\
\hline Human GAPDH & GGAGCGAGATCCCTCCAAAAT & GGCTGTTGTCATACTTCTCATGG \\
\hline Human IL-1 $\beta$ & AACCTCTTCGAGGCACAAGG & GTCCTGGAAGGAGCACTTCAT \\
\hline Human IL-6 & ACCCCCAATAAATATAGGACTGGA & AGAAGGCAACTGGACCGAAG \\
\hline Human IL-10 & TACGGCGCTGTCATCGATTT & TAGAGTCGCCACCCTGATGT \\
\hline Human TNF-a & GCTGCACTITGGAGTGATCG & ATGAGGTACAGGCCCTCTGA \\
\hline Human MCP1 & GATCTCAGTGCAGAGGCTCG & TाTGCTTGTCCAGGTGGTCC \\
\hline Human CXCL1 & CTGGCGGATCCAAGCAAATG & GCCCCTTTGTTCTAAGCCAG \\
\hline Human CXCL10 & GTGGCATTCAAGGAGTACCTC & TGATGGCCTTCGATTCTGGATT \\
\hline Human $\mathrm{COX} 2$ & CTGGCGCTCAGCCATACAG & CGCACTTATACTGGTCAAATCCC \\
\hline Human TF & GCCACCATCTITATCATCCTCC & AGCCTTTCCTCTATGCCAAGC \\
\hline Human TM & AGCGAGGGTAGGGAGGACTTGA & CAGCAGCACTAGGAGGTGAGGT \\
\hline Human EPCR & TTGACGAAGTTTCTGCCGCTAC & CCTGATGCCTCACATGATGGTT \\
\hline Human PAl1 & ACGTGGTTTTCTCACCCTATGG & CATGCCCTTGTCATCAATCTTG \\
\hline
\end{tabular}

Diagnostics, Germany) and SYBR Green PCR Master Mix (Roche Diagnostics, Germany) were applied to detect mRNA expression with primer pair sequences (Table 1).

\section{Lung histology analyses}

Lung tissues were fixed in $4 \%$ paraformaldehyde, dehydrated, embedded, and sectioned at a thickness of $4 \mu \mathrm{m}$ for H\&E staining. A scale of 0 to 3 ( 0 denoted no injury; 1 , mild; 2 , moderate; 3 , severe) was used to evaluate lung injury.

\section{Lung wet/dry weight ratio}

The lungs were weighed immediately after separation and then dried in an incubator at $80^{\circ} \mathrm{C}$ for $48 \mathrm{~h}$ to obtain wet/dry weight ratios.

\section{Lung vascular permeability assessment}

Evans blue dye (EBD) was injected into the tail vein $2 \mathrm{~h}$ before euthanasia. Then the lungs were perfused with PBS and incubated in $1 \mathrm{ml}$ of formamide at $37^{\circ} \mathrm{C}$ for 24 h. Following centrifugation (1000 g for $10 \mathrm{~min}$ ), the EBD in the supernatants was measured spectrophotometrically at $620 \mathrm{~nm}$.

\section{Immunofluorescence staining}

The lung sections were dewaxed and rehydrated, and antigens were retrieved. The primary antibodies were applied at $4{ }^{\circ} \mathrm{C}$ overnight, followed by fluorescent secondary antibody and nuclei staining.

Macrophages or HPMECs were fixed, permeabilized, and blocked. Then the slides were incubated with primary antibodies overnight at $4{ }^{\circ} \mathrm{C}$ followed by 
fluorescent secondary antibody and nuclei staining. Images are representative of three independent experiments.

\section{Statistics}

Statistical analysis was carried out with SPSS software 20.0. The Kaplan-Meier method was used to analyze the survival rate of different groups. Categorized variables were compared by one-way ANOVA or Student's t test. In all tests, $P<0.05$ was considered statistically significant.

\section{Results}

Pharmacological inhibition of pY-STAT3, not total STAT3, improves survival and blunts systemic inflammation and coagulopathy in septic mice

Given the importance of STAT3 in the inflammatory response, we found that the activation of pulmonary STAT3 in CLP-induced septic lung injury mice was significantly increased compared to that in sham group (Fig. 1a). As such, we chose BP-1-102 (BP, a specific STAT3-phospho-Tyr705 inhibitor) and Napabucasin (Na, a total STAT3 inhibitor) to assess the therapeutic potential of STAT3-targeting agents in septic mice. As a result, BP downregulated the elevated pY-STAT3 level in the mouse lung $24 \mathrm{~h}$ after CLP, while Na suppressed STAT3 expression and did not affect the pY-STAT3 level (Fig. 1b). In addition, treatment with BP, but not $\mathrm{Na}$, protected mice against septic death (Fig. 1c), which was associated with reduced systemic proinflammatory and procoagulant-related factors (Fig. 1d). The elevated plasma levels of IL-1 $\beta$, IL-6, TNF- $\alpha$, CXCL10, TF, TAT and D-Dimer in CLP mice were downregulated by BP treatment. However, BP administration had no effect on the level of abnormal plasma plasminogen activator inhibitor 1 (PAI1) level.

\section{pY-STAT3 inhibitor attenuates CLP-induced lung injury in vivo}

To study whether the pY-STAT3 inhibitor BP influences septic lung injury, we checked pulmonary function and some associated indicators in mice. Notably, the septic lung injury features, including an increase in inflammatory cell infiltrates, alveolar septal wall thickening, alveolar congestion and pulmonary edema, were attenuated by BP administration (Fig. 2a). Likewise, the elevated lung injury score (Fig. 2b) and wet/dry weight ratio (Fig. 2c) were reduced in BP-treated group compared with the CLP group. To evaluate pulmonary vascular barrier function, we observed endothelial permeability by an Evans blue dye assay. The results indicated that the CLPinduced lung endothelial hyperpermeability was blunted by $\mathrm{BP}$ treatment (Fig. 2d). Moreover, the immunoblots suggested that BP effectively inhibited CLP-induced pulmonary STAT3 and ERK1/2 phosphorylation (Fig. 2e). RT-PCR analysis indicated that TNF- $\alpha$, IL-6, IL-10, MCP1, CXCL1, CXCL10, COX2, MMP1, MMP9, TF and PAR1 mRNA expression levels were increased in lung tissues during CLP compared with the sham group but decreased under BP administration except for IL-1 $\beta$, ICAM1 and MMP8 mRNA levels (Fig. 2f). In addition, the images of TF, PAR1 and MMP9 fluorescent staining also partly verified the results of RT-PCR analysis (Fig. $2 \mathrm{~g}$, $\mathrm{h}$ and i). As macrophages play a vital part in the pathogenesis of

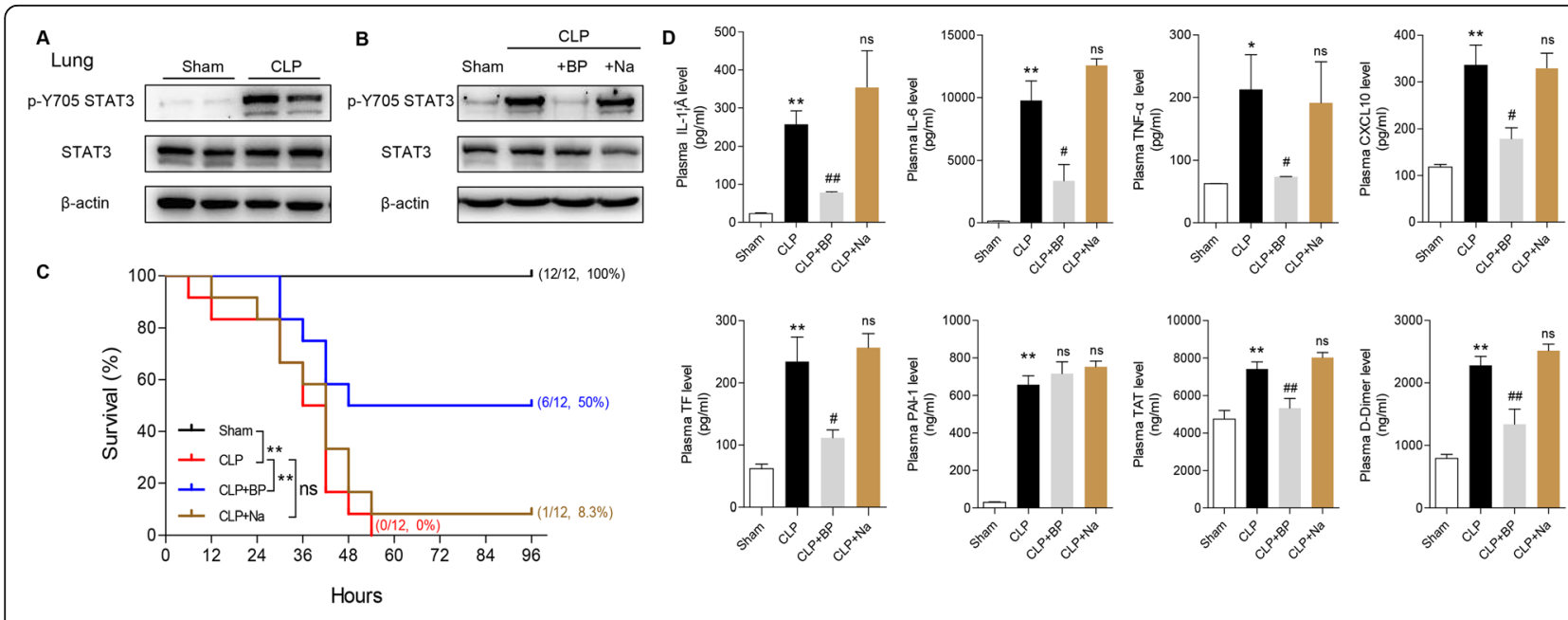

Fig. $1 \mathrm{BP}$ but not Na, protects mice from septic death, systemic inflammation and coagulopathy. a Immunoblotting analysis of pulmonary STAT3 and phospho-Y705-STAT3 in mice $24 \mathrm{~h}$ after CLP or shame operation. $\mathbf{b}$ Immunoblotting analysis of pulmonary STAT3 and pY-STAT3 in mice $24 \mathrm{~h}$ after CLP with BP or Na administration ( $n=3$ per group). c Administration of BP, but not Na prevented CLP-induced mouse death ( $n=12$ mice per group; Kaplan-Meier survival analysis). d IL-13, IL-6, TNF-a, CXCL10, TF, PAl1, TAT and D-Dimer concentrations in plasma were measured $24 \mathrm{~h}$ after the CLP procedure by ELISA ( $n \geq 5$ per group; two-tailed Student's test). ${ }^{*} P<0.01$ and ${ }^{*} P<0.05$ (CLP versus Sham); \#\#P<0.01 and \#P<0.05 (CLP versus CLP + BP); ns indicates not significant 


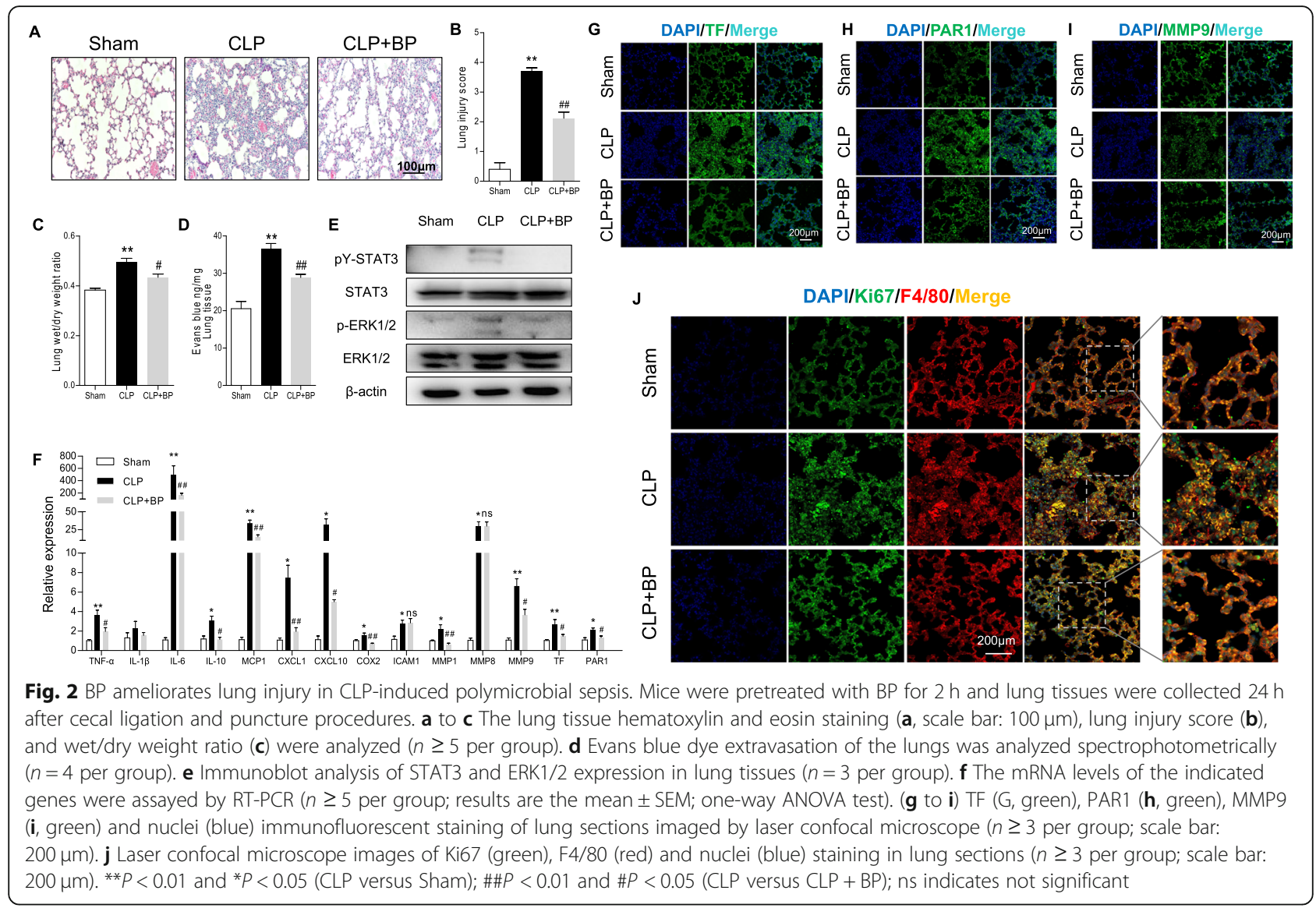

sepsis, we performed F4/80 labeling for macrophages and Ki67 labeling for proliferating cells, which illustrated that the increase in macrophages infiltration in septic lung tissues was effectively decreased by BP treatment (Fig. 2j).

\section{pY-STAT3 inhibitor protects HPMECs from LPS damage}

The vascular system dysfunction characteristic of endothelial hyperpermeability is a key component in sepsis, so we investigated the effect of $\mathrm{BP}$ on endothelial cell function under LPS challenge. First, we tested the CCK8 level of HPMECs with BP and determined that $5 \mu \mathrm{M}$ was an ideal concentration (Fig. 3a). Then, we measured the HPMECs proliferation and found that LPS-induced cell proliferation was inhibited by BP (Fig. 3b). The tube formation assay indicated that the network formation of HPMECs was significantly enhanced under LPS stimulation but restrained by BP (Fig. 3c). To address whether pY-STAT3 inhibition has an effect on the regulation of vascular integrity with LPS, we evaluated HPMECs monolayer permeability and found that BP partly curbed the increase in HPMECs permeability induced by LPS (Fig. 3d). Since endothelial permeability is related to the control of cohesion and to the organization of intercellular junctions, we further examined the protein expression of junction proteins, including VE-cadherin and $\alpha$ -
E-catenin, through ELISA and immunoblots. Interestingly, neither LPS nor BP affected on these two indicators in the cell supernatant and lysate (Fig. 3e and f). However, the integrity of VE-cadherin and $\alpha$-E-catenin detected by immunofluorescence staining was disrupted and fractured between cells that were repaired by BP administration (Fig. 3g). This meant that LPS and BP did not induce changes in VE-cadherin and $\alpha$-E-catenin protein expression but affected the localization of these proteins. To establish whether the hyperpermeability was indirectly due to the elevation in cytokines, we transferred LPStreated HPMECs-conditioned medium (CM) and LPS + BP CM to newly cultured HPMECs. The results showed that LPS-treated CM led to more severe endothelial leakage than LPS + BP CM in HPMECs (Fig. 3h). Next, we measured the mRNA expression of proinflammatory mediators, including TNF- $\alpha$, IL-1 $\beta$, IL-6, IL-10, MCP1, CXCL1, CXCL10 and COX2, and coagulation-associated factors, including TF, thrombomodulin (TM), endothelial protein $C$ receptor (EPCR) and PAI1. It showed that BP administration suppressed the LPS challenge-induced increase in the expression of most proinflammatory mediators except for that of CXCL1 and COX2, but had no effect on the four abnormal coagulation associated factors (Fig. 3i). Activation of inflammation signaling, such as the 


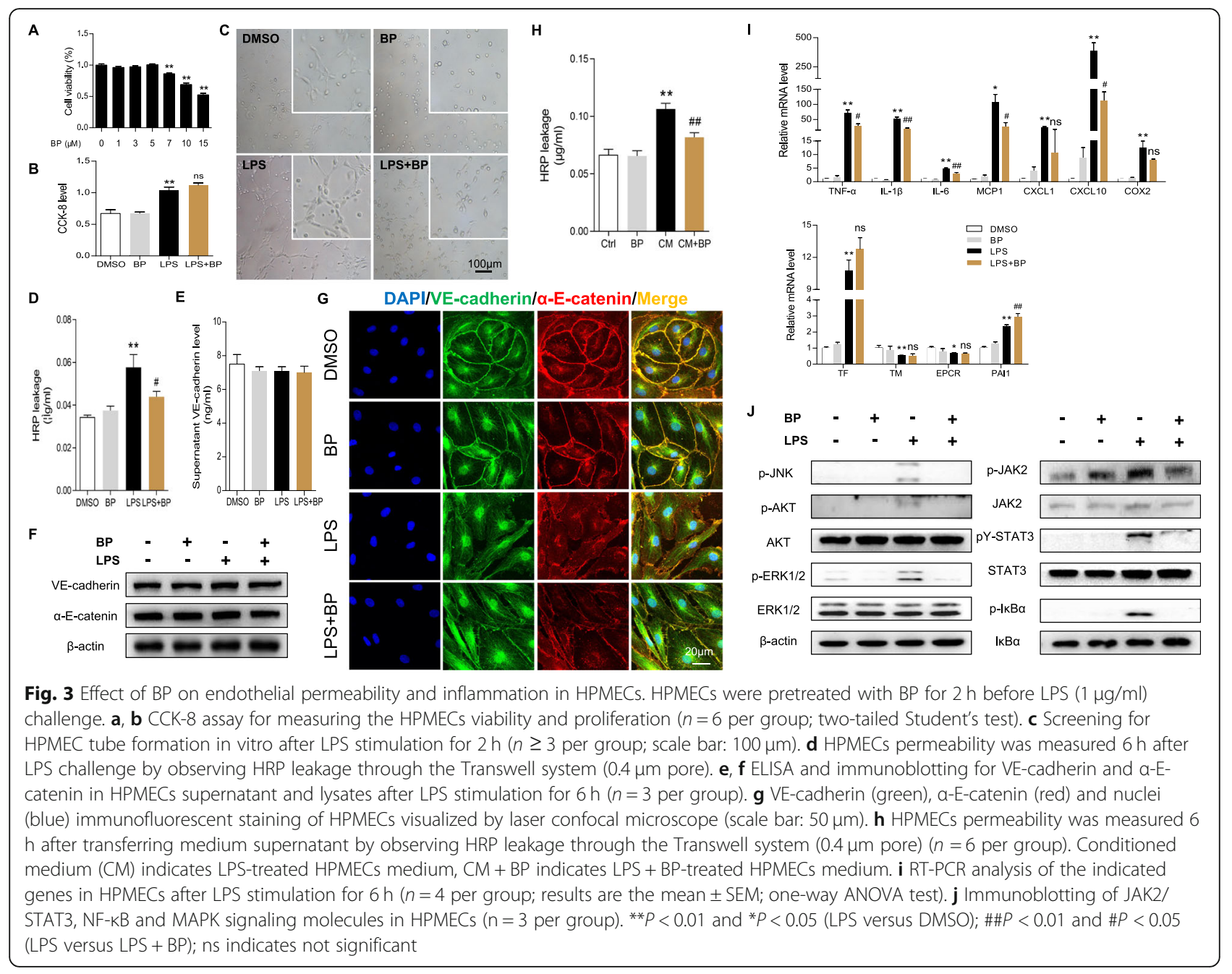

JAK2/STAT3, NF-кB, MAPK and AKT pathways, is necessary for TLR-mediated cytokine production. We evaluated JAK2/STAT3 pathway activation by measuring the phosphorylation of STAT3 and JAK2, NF-kB pathway activation by measuring the phosphorylation of IKB $\alpha$, MAPK pathway activation by measuring the phosphorylation of ERK and JNK, and AKT pathway activation by measuring the phosphorylation of AKT and found that the phosphorylation of all these proteins was decreased by BP treatment in LPS-stimulated HPMECs (Fig. 3j). In summary, these findings collectively demonstrated that pY-STAT3 pharmacological inhibition prevented HPMECs from LPS damage through the JAK2/STAT3, NF- $k$ B, MAPK and AKT pathways and enhanced endothelial integrity by cementing VE-cadherin and $\alpha$-E-catenin localization and reducing cytokine levels.

PY-STAT3 inhibitor reduces the surge in inflammatory mediators and TF in macrophages stimulated by LPS In light of the decrease in pulmonary macrophage infiltration, we aimed to explore the role of BP in activated macrophage regulation. Similarly, we tested the CCK-8 level of Raw264.7 cells with BP and chose $5 \mu \mathrm{M}$ as the final concentration (Fig. 4a). As a result, LPS-induced macrophage proliferation was obviously inhibited by BP (Fig. 4b). The IL-6, TNF- $\alpha$ and TF levles in the cell supernatant were increased by LPS stimulation and diminished by BP treatment (Fig. 4c). The fluorescence intensity of TF in macrophages was enhanced by LPS induction but weakened in the presence of BP (Fig. 4d). We next examined the mRNA expression of TNF- $\alpha$, IL-1 $\beta$, IL-6, IL-10, MCP1, CXCL1, CXCL10, CXCL16, COX2, MMP1, MMP9, TF and PAI1, which suggested a similar trend to previous pulmonary results (Fig. 4e). Since the JAK2/STAT3, NF-kB, MAPK and AKT pathways are involved in activation of inflammation response and coagulation, we found that the LPSchallenged hyperactivation of JAK2/STAT3, NF- $\mathrm{kB}$ and MAPK pathways in macrophages was efficiently controlled by BP administration (Fig. 4f). Therefore, these results demonstrated that the pY-STAT3 inhibitor downregulated macrophage cytokine and TF production by the JAK2/ STAT3, NF-kB, MAPK and AKT pathways. 


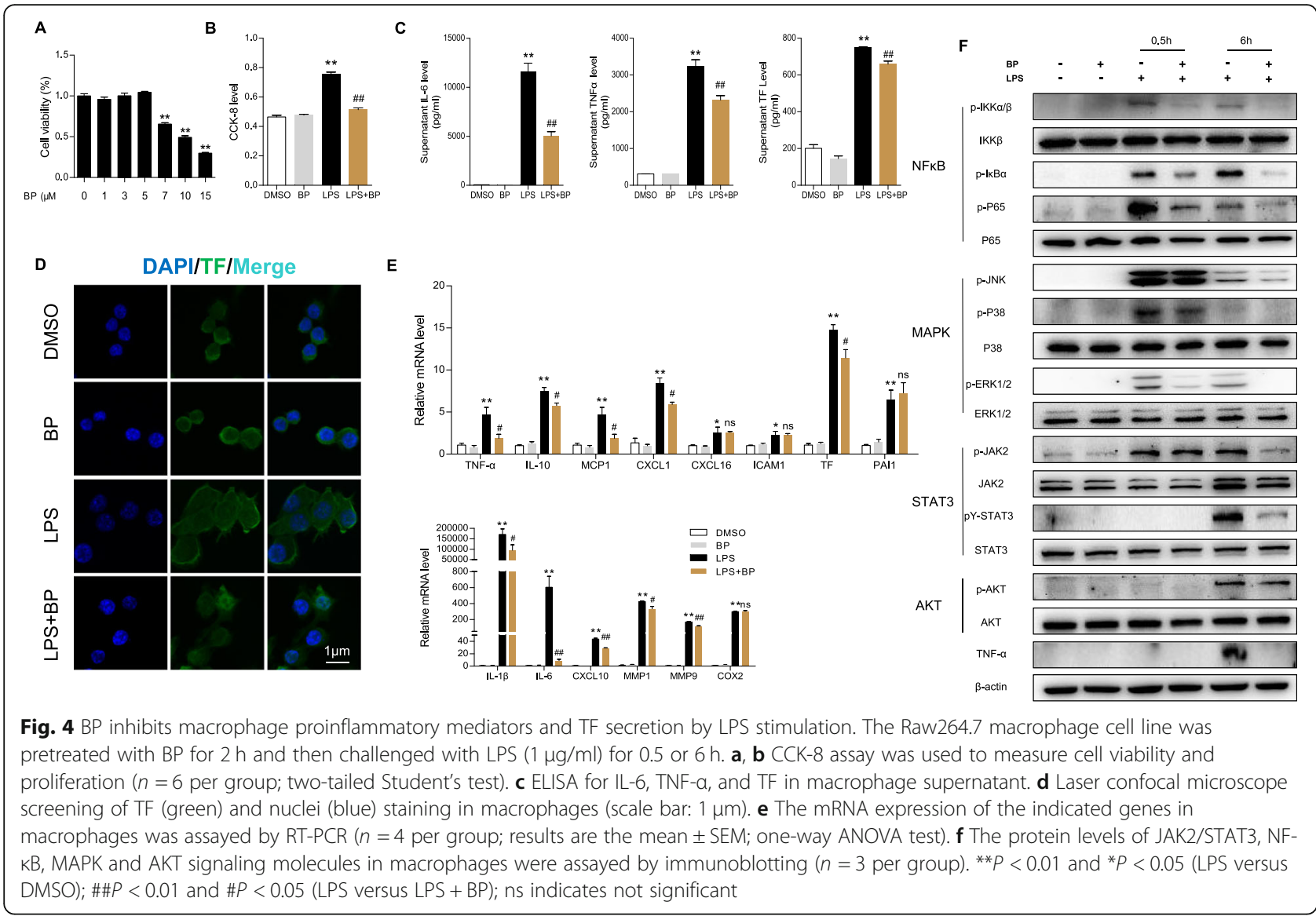

\section{Genetic suppression of pY-STAT3 limits the LPS-induced hyperinflammatory response in macrophages}

To rule out side effects of the inhibitor, we genetically suppressed pY-STAT3 through the Y705-STAT3 mutation. As expected, the LPS-induced proliferation of pYSTAT3 mutant macrophages was successfully limited (Fig. 5a). We found decreased secretion of cell supernatant IL-6, TNF- $\alpha$ and TF in the cell supernatants of LPSstimulated pY-STAT3 mutant macrophages compared with LPS-stimulated control macrophages (Fig. 5B). Similarly, the fluorescence intensity of TF in LPS-challenged pY-STAT3 mutant macrophages was reduced (Fig. 5c). In addition, RT-PCR analysis revealed a decline in the mRNA expression of TNF- $\alpha$, IL-1 $\beta$, IL-6, IL-10, MCP1, CXCL1, CXCL10, MMP9 and TF in LPS-stimulated pYSTAT3 mutant macrophages (Fig. 5d). We also found that the hyperactivation of the JAK2/STAT3, NF-kB, MAPK and AKT pathways in pY-STAT3 mutant macrophages challenged by LPS was effectively curbed (Fig. 5e). In short, these findings verified that the targeted suppression of pY-STAT3 is capable of modulating the macrophage inflammatory response and TF expression via the JAK2/STAT3, NF-кB, MAPK and AKT signaling pathways.

\section{TLR4 modulates pY-STAT3-associated inflammatory responses in vitro and in vivo}

Based on our results that pY-STAT3 was related to the NF- $\mathrm{kB}$ pathway in macrophages, we hypothesized that there is crosstalk between TLR4 and STAT3 signaling. We next extracted peritoneal macrophages (PMs) from TLR4 mutant mice. Upon LPS stimulation, TLR4 mutant PMs expressed obviously lower mRNA levels of inflammatory mediators (TNF- $\alpha$, IL-1 $\beta$, IL-6, IL-10 and CXCL1), TF and PAR1 (Fig. 6a). The immunoblot revealed a weaker activation of STAT3 in LPS-challenged TLR4 mutant PMs (Fig. 6b). These proved a crosstalk between TLR4 and STAT3 pathway in LPS-induced macrophages. We also explored the effects of CLP in TLR4 mutant mice and found that TLR4 mutant CLP mice had milder lung injury than wild type CLP mice (Fig. 6c, d). The immunoblots suggested that TLR4 mutant CLP mice had lower levels of pY-STAT3 in lung tissue (Fig. 6e). In addition, the mutation of TLR4 prolonged animal survival in the CLP model (Fig. 6f).

\section{Discussion}

Sepsis ia a serious problem worldwide, incurring the greatest hospital costs to hospital and giving rise to most 


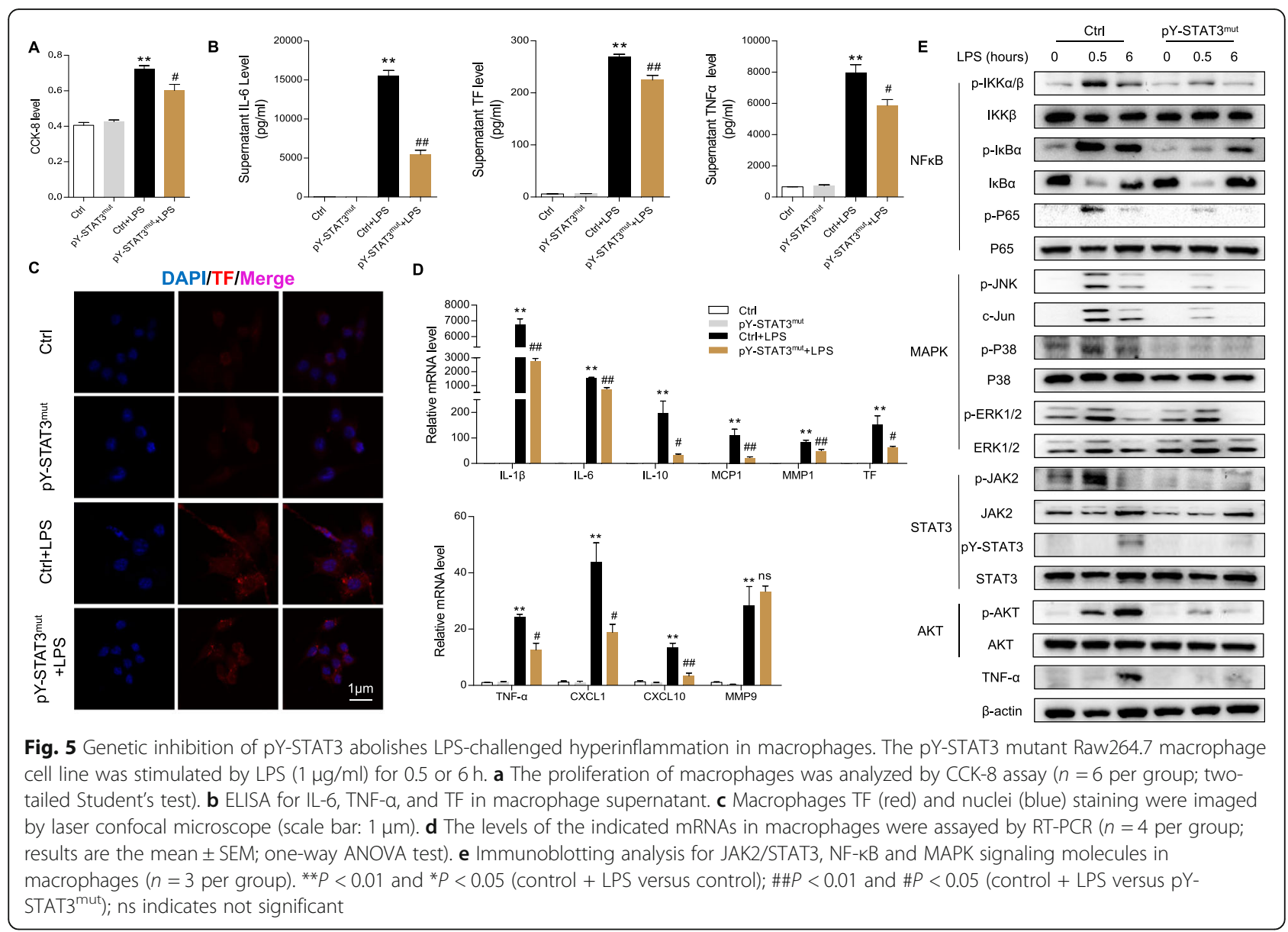

deaths in intensive care units [33]. Clearly, the activation of pattern recognition receptors (PPRs) in response to microbial pathogens triggers the imbalance of the inflammation-coagulation network, leading to death during sepsis [34]. Consequently, elucidating the key signaling molecules of this network can provide a promising therapeutic strategy for sepsis. Our findings demonstrated that pY-STAT3 is an effective therapeutic target in regulating inflammation and coagulation activation, which contributes to the pathogenesis of sepsis.

STAT3 is a pleotropic transcription factor that mediates multiple biological activities, including inflammation, metabolism and development [35]. STAT3 has previously been shown to be activated by phosphorylation at tyrosine 705 and serine 727 following stimulation by TLR agonists, cytokines and growth factors [36]. Our studies found that STAT3 was hyperactive at tyrosine 705 in the lung tissues of CLP-induced septic mice. Previous studies have reported that STAT3 inhibitors have effects on cancers, such as pancreas and esophagus cancers [37]. Therefore, the total STAT3 inhibitor Na and the STAT3 phospho-Tyr705 selective inhibitor BP were chosen as treatments for CLP mice. The results showed that $\mathrm{BP}$, but not $\mathrm{Na}$, efficaciously protected mice against septic fatal death, marked inflammation and defective coagulation which indicated the potential role of pY-STAT3 in sepsis. Since STAT3-deficient mice are embryonic lethal, conditional tissue-specific gene knockout strategies were applied for STAT3 study in different diseases. Macrophage/neutrophil-specific STAT3 deletion mice were more susceptible to endotoxemia and sepsis associated with higher systemic inflammation, weaker bacterial clearance, more severe multiple organ dysfunction and increased mortality [30]. Our findings also verified that the total STAT3 inhibitor could not rescue the mice from septic death.

Coagulopathy is a critical host response to infection accompanied by an inflammatory response that can result in disseminated intravascular coagulation (DIC) with increased mortality during sepsis [38]. DIC, featured by the systemic overactivation of coagulation, is associated with intravascular thrombosis formation that subsequently leads to multiple organ dysfunction $[8,18]$. The products and stimuli from microbes induce TF secretion, a potent initiated thrombin activator mainly produced by monocytes, epithelial cells and endothelial cells, to promote septic coagulopathy, followed by increasing the plasma levels of TAT, D-Dimer and PAI1 


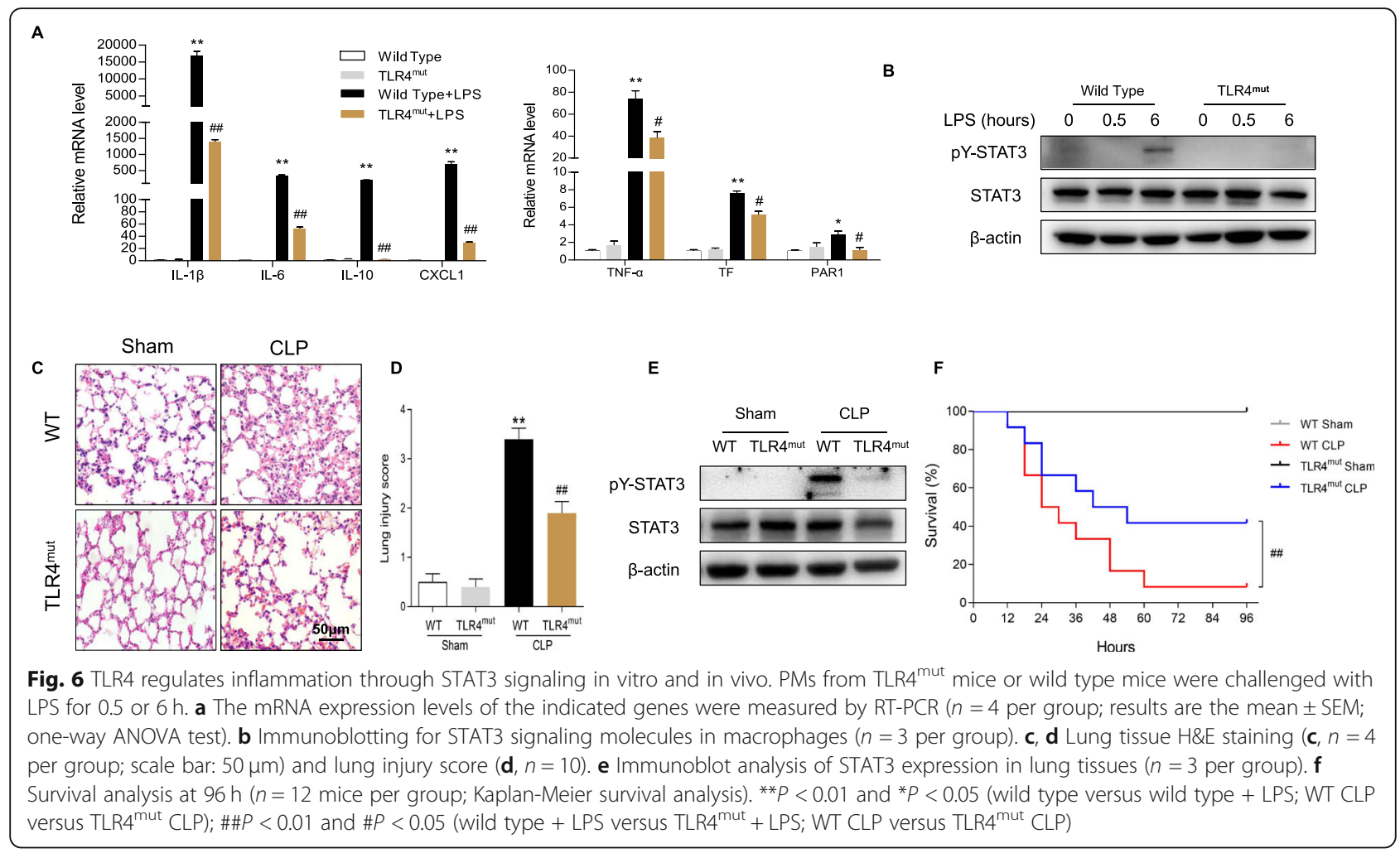

$[39,40]$. Our results suggested that pY-STAT3 inhibition suppressed the production of TF, TAT and D-Dimer in septic mice. However, the increased PAI1 level was not affected by the treatment. We hypothesized that interference of pY-STAT3 had no effect on the regulation of the fibrinolytic system.

ALI is one of the most important organ injuries caused by sepsis and has complex mechanisms, including cytokine storm and pulmonary capillary leakage [41-43]. Previous studies have shown that Stattic and LLL12 were utilized to suppress STAT3 activity and ameliorate inflammatory responses in LPS-induced ALI and LPSchallenged macrophages [29]. Our results also supported the notion that the pY-STAT3 inhibitor BP had obvious effects on pulmonary protection. Vascular inflammation and hyperpermeability are common features of the pathogenesis of sepsis, and pulmonary endothelial cell dysfunction plays a key part in sepsis-induced ALI [44-46]. Numerous studies have shown that STAT3 signaling is involved in endothelial cell function [47-49]. However, the detailed mechanism of cell permeability is still unclear. In the present study, we revealed that $\mathrm{BP}$ administration stabilized HPMECs integrity by affecting the localization of VE-cadherin and $\alpha$-E-catenin. Angiogenesis which indicates the imbalance of endothelial cells, was impaired by BP under LPS stimulation. We found that the LPSinduced HPMECs tube formation was restrained by pYSTAT3 inhibition. Coagulation activation-associated factors are also closely related to endothelial cell damage including TF, TM, EPCR and PAI1. Previous studies have found that the targeted therapies for these coagulation indicators had protective effects on sepsis $[4,6]$. Nevertheless, our study discovered that the pY-STAT3 inhibitor BP could not improve the abnormal TF, TM, EPCR and PAI1 levels in HPMECs by LPS priming.

The abnormal changes in the cellular biological characteristics of the inflammatory response and cell growth exhibit homeostatic imbalances during sepsis [50]. We found that the proinflammatory factor production and cell proliferation were suppressed by pY-STAT3 inhibition in vivo and in vitro. The proliferation of macrophages stimulated by LPS was controlled by pharmacological or genetic pY-STAT3 inhibition. The immunofluorescence double staining with Ki67 and F4/ 80 for proliferating macrophages showed that the increased lung macrophage infiltration in CLP mice was effectively restrained by $\mathrm{BP}$ treatment.

The IL-6/JAK2/STAT3, NF-кB, MAPK and AKT pathways are central signaling pathways in pathophysiological processes and interact with each other in inflammatory diseases [22, 51, 52]. However, the crosstalk between these signaling pathways in sepsis is not fully understood. Our results proved that the inhibition of STAT3 activation can decrease hyperinflammatory factor production in cell and animal experiments through the JAK2/STAT3 (p-JAK2 and pY-STAT3), NF- 
$\kappa B(p-I K K \alpha / \beta, p-I \kappa B \alpha$ and p-P65), MAPK (p-JNK, pJun, p-P38 and p-ERK1/2) and AKT (p-AKT) pathways in an interactive way. The TF gene expression by LPS induction in macrophages and endothelial cells requires the activation of various transcription factors, including NF- $\mathrm{BB}$, MAPK, Egr-1 and protease activated receptors (PARs) [53-56]. In addition, to our knowledge, our study is the first to find that TF expression is regulated by STAT3 signaling with LPS stimulation, which may provide a new molecular mechanism for the diseases mediated by TF. To verify whether STAT3 has a connection with TLR4, we extracted PMs from TLR4 mutant mice and found that the TLR4-mutant macrophages showed reduced activation of STAT3 and production of inflammatory and coagulant factors following LPS challenge. Moreover, we also explored the effects of CLP on TLR4 mutant mice and found that TLR4 mutant CLP mice have milder lung injury, lower STAT3 activation and a higher survival rate than wild type CLP mice.
While our results showed that pY-STAT3 is a promising therapeutic target in sepsis, the role of STAT3 as an essential transcription factor makes the development of pSTAT3-targeted therapies challenging. Further indepth analysis of the regulatory function of the STAT3 pathway in sepsis will be the focus of future research. We should also focus on the role of negative regulators of the STAT3 pathway in the treatment of sepsis.

\section{Conclusions}

In summary, as shown in Fig. 7, our study revealed the crucial role of activated STAT3 in inflammation and coagulation in sepsis. The inhibition of STAT3 activation can markedly limit the hyperactivation of the inflammatory response, suppress the coagulation activation, and protect the endothelial barrier function through the MAPK, AKT, STAT3 and NF- $k B$ pathways which promote each other interactively. Therefore, targeting $\mathrm{pY}$ STAT3 presents a potential therapeutic strategy for combating sepsis.
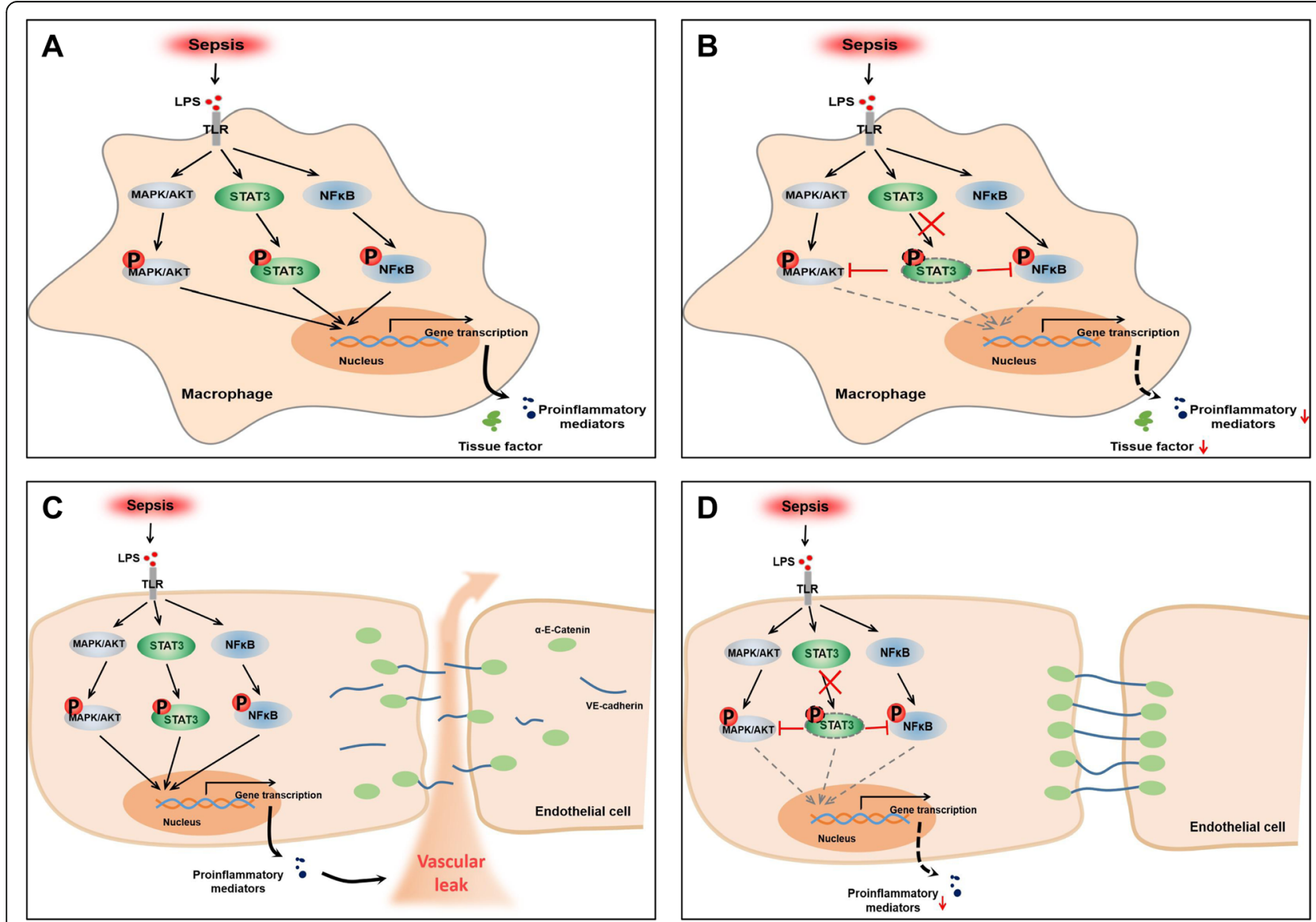

Fig. 7 Schematic of the regulation of septic inflammation and coagulopathy in endothelial cells and macrophages by STAT3 signaling. $\mathbf{a}, \mathbf{b}$ In macrophages, inhibition of pY-STAT3 reduces sepsis-induced proinflammatory mediators and TF expression through inactivation of JAK2/STAT3, NF-KB, MAPK and AKT signaling. $\mathbf{c}$, $\mathbf{d}$ In endothelial cells, inhibition of pY-STAT3 protects cells from inflammatory injury and hyperpermeability via JAK2/STAT3, NF-KB, MAPK and AKT pathways and enhances cellular VE-cadherin/a-E-catenin localization 


\section{Supplementary information}

Supplementary information accompanies this paper at https://doi.org/10. 1186/s12964-020-00603-z.

\section{Additional file 1.}

\section{Abbreviations}

pY-STAT3: phospho-Tyr705 of STAT3; CLP: Cecal ligation and puncture; BP: BP-1-102; Na: Napabucasin; HPMECs: Human pulmonary microvascular endothelial cells; LPS: Lipopolysaccharide; TF: Tissue factor; TAT: Thrombinantithrombin complex; ALI: Acute lung injury; TLR4: Toll-like receptor 4; PMs: Peritoneal macrophages; CCK-8: Cell Counting Kit-8; PAI1: Plasminogen activator inhibitor 1; EBD: Evans blue dye; TM: Thrombomodulin; EPCR: Endothelial protein C receptor; PPRs: Pattern recognition receptors: DIC: Disseminated intravascular coagulation; PARs: Protease activated receptors

\section{Acknowledgements}

Not applicable.

\section{Authors' contributions}

SX, performed the experiments and wrote the paper; XP, LM, HP and Y Hu collected and analyzed the data; $X Y, Z C$ and $W X$ designed the figure legends; $Y Y$ and $Y H$ contributed to the drafting of the manuscript; JP conceived the study. All authors read and approved the final manuscript.

\section{Funding}

This work was supported by the National Natural Science Foundation of China (81873949, 81671968 and 8197894), the Natural Science Foundation of Zhejiang Province of China (LY17H150003), the medical innovation discipline of Zhejiang Province of China (critical care medicine, Y2015), the medical and health research project of Zhejiang Province of China (WKJ-ZJ-1909), the project of Wenzhou science and technology (2018ZY002).

\section{Availability of data and materials}

All data generated or analysed during this study are included in this published article.

\section{Ethics approval and consent to participate}

All mice were maintained in a pathogen-free facility and received food and water ad lib. Animal care and experimentation were conducted with approval from Wenzhou Medical University Institutional Animal Care and Use Committees.

\section{Consent for publication}

All authors have read and approved the final manuscript.

\section{Competing interests}

The authors declare that they have no competing interests.

\section{Author details}

'Department of Intensive Care Unit, The First Affiliated Hospital of Wenzhou Medical University, Nanbaixiang road, Wenzhou, Zhejiang 325000, P.R. China. ${ }^{2}$ Wenzhou Medical University, Wenzhou, Zhejiang, P.R. China. ${ }^{3}$ Department of Blood Transfusion, The First Affiliated Hospital of Wenzhou Medical University, Wenzhou, Zhejiang, P.R. China.

Received: 25 September 2019 Accepted: 26 May 2020

Published online: 08 July 2020

References

1. Singer $\mathrm{M}$, et al. The third international consensus definitions for sepsis and septic shock (Sepsis-3). JAMA. 2016;315(8):801-10.

2. Seymour CW, et al. Assessment of clinical criteria for sepsis: for the third international consensus definitions for sepsis and septic shock (Sepsis-3). JAMA. 2016;315(8):762-74.

3. Angus DC, van der Poll T. Severe sepsis and septic shock. N Engl J Med. 2013;369(9):840-51.

4. Fink MP, Warren HS. Strategies to improve drug development for sepsis. Nat Rev Drug Discov. 2014;13(10):58.
5. Hotchkiss RS, Sherwood ER. Immunology. Getting sepsis therapy right. Science. 2015:347(6227):1201-2.

6. van der Poll T, et al. The immunopathology of sepsis and potential therapeutic targets. Nat Rev Immunol. 2017:17(7):407-20.

7. Venet F, Monneret G. Advances in the understanding and treatment of sepsis-induced immunosuppression. Nat Rev Nephrol. 2018;14(2):121-37.

8. Levi M, van der Poll T. Coagulation and sepsis. Thromb Res. 2017;149:38-44.

9. Levi M, van der Poll T, Buller HR. Bidirectional relation between inflammation and coagulation. Circulation. 2004;109(22):2698-704.

10. Wang $Y$, et al. Monocytes regulate systemic coagulation and inflammation in abdominal sepsis. Am J Physiol Heart Circ Physiol. 2015;308(5):H540-7.

11. Chen $\mathrm{Q}$, et al. Increased gene copy number of DEFA1/DEFA3 worsens sepsis by inducing endothelial pyroptosis. Proc Natl Acad Sci U S A. 2019; 116(8):3161-70.

12. Moussa MD, et al. Evaluation of endothelial damage in sepsis-related ARDS using circulating endothelial cells. Intensive Care Med. 2015;41(2):231-8.

13. Wu $\mathrm{C}$, et al. Inflammasome activation triggers blood clotting and host death through Pyroptosis. Immunity. 2019;50(6):1401-11 e4.

14. Scully M, Levi M. How we manage haemostasis during sepsis. $\mathrm{Br} \mathrm{J}$ Haematol. 2019;185(2):209-18.

15. Burzynski LC, et al. The coagulation and immune systems are directly linked through the activation of interleukin-1alpha by thrombin. Immunity. 2019; 50(4):1033-42 e6.

16. Jackson SP, Darbousset R, Schoenwaelder SM. Thromboinflammation: challenges of therapeutically targeting coagulation and other host defense mechanisms. Blood. 2019;133(9):906-18.

17. Tucker El, et al. Inhibition of factor XI activation attenuates inflammation and coagulopathy while improving the survival of mouse polymicrobial sepsis. Blood. 2012;119(20):4762-8.

18. Gando S, Levi M, Toh CH. Disseminated intravascular coagulation. Nat Rev Dis Primers. 2016;2:16037.

19. Alphonsus CS, Rodseth RN. The endothelial glycocalyx: a review of the vascular barrier. Anaesthesia. 2014;69(7):777-84.

20. Grewal PK, et al. The Ashwell receptor mitigates the lethal coagulopathy of sepsis. Nat Med. 2008;14(6):648-55.

21. Popescu NI, et al. Peptidoglycan induces disseminated intravascular coagulation in baboons through activation of both coagulation pathways. Blood. 2018;132(8):849-60.

22. Garbers C, et al. Interleukin-6: designing specific therapeutics for a complex cytokine. Nat Rev Drug Discov. 2018:17(6):395-412.

23. Kang $\mathrm{S}$, et al. Implications of IL-6 targeting therapy for sepsis. mmunotherapy. 2017:3·2

24. Alsaffar $\mathrm{H}$, et al. Interleukin-6 promotes a sustained loss of endothelial barrier function via Janus kinase-mediated STAT3 phosphorylation and de novo protein synthesis. Am J Phys Cell Phys. 2018;314(5):C589-602.

25. Cai $B$, et al. The specific roles of JAK/STAT signaling pathway in sepsis. Inflammation. 2015:38(4):1599-608.

26. Hui L, et al. Inhibition of Janus kinase 2 and signal transduction and activator of transcription 3 protect against cecal ligation and punctureinduced multiple organ damage and mortality. J Trauma. 2009;66(3):859-65.

27. Peña $\mathrm{G}$, et al. JAK2 inhibition prevents innate immune responses and rescues animals from sepsis. J Mol Med. 2010;88(8):851-9.

28. Peña $\mathrm{G}$, et al. Unphosphorylated STAT3 modulates alpha7 nicotinic receptor signaling and cytokine production in sepsis. Eur J Immunol. 2010;40(9): 2580-9.

29. Zhao J, et al. Protective effect of suppressing STAT3 activity in LPS-induced acute lung injury. Am J Phys Lung Cell Mol Phys. 2016;311(5):L868-80.

30. Matsukawa A, et al. Aberrant inflammation and lethality to septic peritonitis in mice lacking STAT3 in macrophages and neutrophils. J Immunol. 2003; 171(11):6198-205

31. Kiyoshi Takeda BEC, Kaisho T, Tsujimura T, Terada N, Förster I, Akira S, et al. Immunity. 1999;10(1):11.

32. Xu S, et al. BMSCs ameliorate septic coagulopathy by suppressing inflammation in cecal ligation and puncture-induced sepsis. J Cell Sci. 2018; 131:3

33. Fleischmann-Struzek C, et al. Challenges in assessing the burden of sepsis and understanding the inequalities of sepsis outcomes between National Health Systems: secular trends in sepsis and infection incidence and mortality in Germany. Intensive Care Med. 2018;44(11):1826-35.

34. Broz P, Monack DM. Newly described pattern recognition receptors team up against intracellular pathogens. Nat Rev Immunol. 2013;13(8):551-65. 
35. Stark GR, Darnell JE Jr. The JAK-STAT pathway at twenty. Immunity. 2012; 36(4):503-14.

36. Yue $\mathrm{H}$, et al. Role of nuclear unphosphorylated STAT3 in angiotensin II type 1 receptor-induced cardiac hypertrophy. Cardiovasc Res. 2010;85(1):90-9.

37. Johnson DE, O'Keefe RA, Grandis JR. Targeting the IL-6/JAK/STAT3 signalling axis in cancer. Nat Rev Clin Oncol. 2018;15(4):234-48.

38. Iba T, et al. Advance in the Management of Sepsis-Induced Coagulopathy and Disseminated Intravascular Coagulation. J Clin Med. 2019:8:5.

39. Kansuke Koyama SM, Nunomiya S, Koinuma T, Wada M, Sakata A, Ohmori T, et al. Combination of thrombin-antithrombin complex, plasminogen activator inhibitor-1, and protein C activity for early identification of severe coagulopathy in initial phase of sepsis: a prospective observational study. Crit Care. 2014;18(1):11

40. Satoshi Gando DS, Ishikura H, Ueyama M. A randomized, controlled, multicenter trial of the effects of antithrombin on disseminated intravascular coagulation in patients with sepsis. Crit Care. 2013;17:6.

41. Gong H, et al. HIF2alpha signaling inhibits adherens junctional disruption in acute lung injury. J Clin Invest. 2015;125(2):652-64.

42. London NR, Zhu W, Bozza FA, Smith MCP, Greif DM, Sorensen LK, et al. Targeting Robo4-dependent slit signaling to survive the cytokine storm in sepsis and influenza. Sci Transl Med. 2018;2:23.

43. Lelubre C, Vincent JL. Mechanisms and treatment of organ failure in sepsis. Nat Rev Nephrol. 2018;14(7):417-27.

44. Opal SM, van der Poll T. Endothelial barrier dysfunction in septic shock. Intern Med. 2015;277(3):277-93.

45. Rizzo AN, et al. Targeting Abl kinases to regulate vascular leak during sepsis and acute respiratory distress syndrome. Arterioscler Thromb Vasc Biol. 2015;35(5):1071-9.

46. Goldenberg NM, Steinberg BE, Slutsky AS, Lee WL. Broken barriers: a new take on sepsis pathogenesis. Sci Transl Med. 2011;3:88).

47. Schnoor $\mathrm{M}$, et al. Actin dynamics in the regulation of endothelial barrier functions and neutrophil recruitment during endotoxemia and sepsis. Cell Mol Life Sci. 2017:74(11):1985-97.

48. Wei LH, et al. The role of IL-6 trans-signaling in vascular leakage: implications for ovarian hyperstimulation syndrome in a murine model. J Clin Endocrinol Metab. 2013;98(3):E472-84.

49. Yun JH, et al. Endothelial STAT3 activation increases vascular leakage through downregulating tight junction proteins: implications for diabetic retinopathy. J Cell Physiol. 2017;232(5):1123-34.

50. Li X, et al. O-GlcNAc transferase suppresses inflammation and necroptosis by targeting receptor-interacting serine/threonine-protein kinase 3 . Immunity. 2019;50(3):576-90 e6.

51. Greenhill CJ, et al. IL-6 trans-signaling modulates TLR4-dependent inflammatory responses via STAT3. J Immunol. 2011;186(2):1199-208.

52. Li X, et al. Myeloid-derived cullin 3 promotes STAT3 phosphorylation by inhibiting OGT expression and protects against intestinal inflammation. J Exp Med. 2017;214(4):1093-109.

53. Bode $\mathrm{M}$, Mackman N. Regulation of tissue factor gene expression in monocytes and endothelial cells: thromboxane A2 as a new player. Vasc Pharmacol. 2014;62(2):57-62.

54. Dauphinee SM, Karsan A. Lipopolysaccharide signaling in endothelial cells. Lab Investig. 2006;86(1):9-22.

55. Lee R, Williams JC, Mackman N. P2X7 regulation of macrophage tissue factor activity and microparticle generation. J Thromb Haemost. 2012;10(9): 1965-7.

56. Reinhardt C, et al. Tissue factor and PAR1 promote microbiota-induced intestinal vascular remodelling. Nature. 2012;483(7391):627-31.

\section{Publisher's Note}

Springer Nature remains neutral with regard to jurisdictional claims in published maps and institutional affiliations.

\section{Ready to submit your research? Choose BMC and benefit from:}

- fast, convenient online submission

- thorough peer review by experienced researchers in your field

- rapid publication on acceptance

- support for research data, including large and complex data types

- gold Open Access which fosters wider collaboration and increased citations

- maximum visibility for your research: over $100 \mathrm{M}$ website views per year

At $\mathrm{BMC}$, research is always in progress.

Learn more biomedcentral.com/submissions 